with acid erosion in caries free mouths. My belief is that a large cause of this is the fact that families are not registering fruit juice, yoghurts and 'smoothies' as a source of both sucrose and acid. These products are often given more than once a day by thoughtful caring parents in

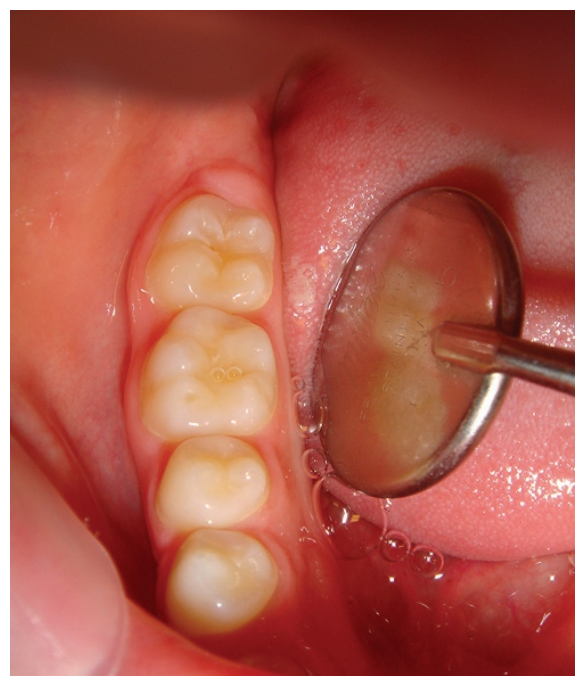

Fig. 1 One circle (MB cusp of LR6)

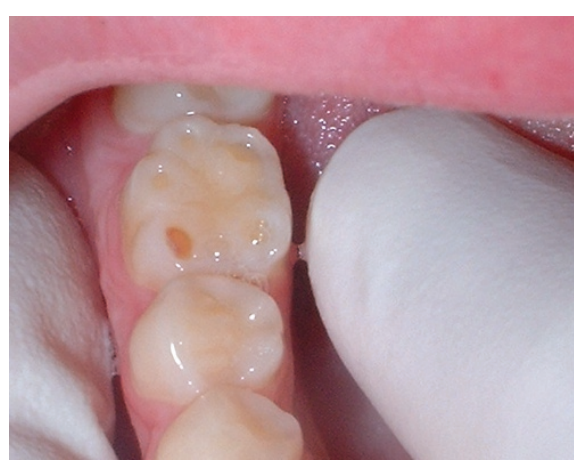

Fig. 2 Five circles (three buccal, two lingual cusps, eroded LR6)

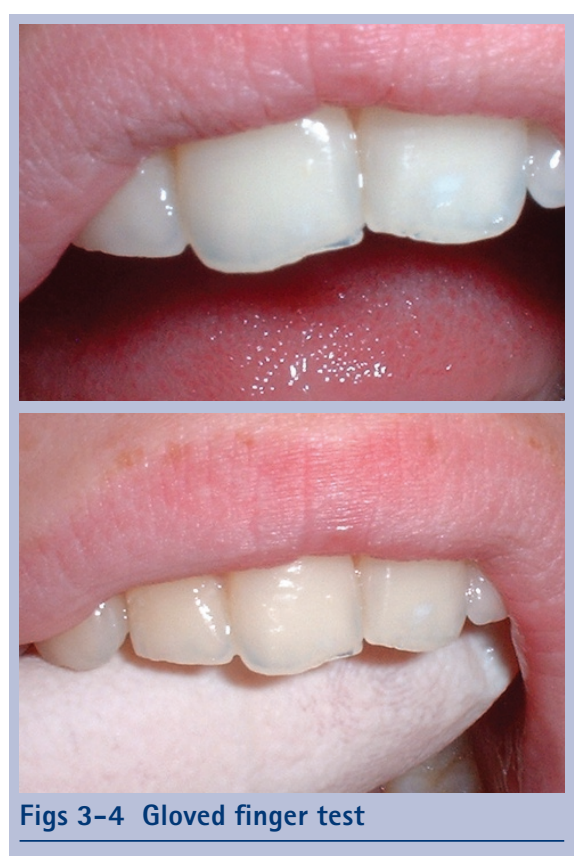

the belief that the products are 'healthy' and they miss the fact that the products are vehicles of fermentable sugars and acids. As the mouths are often caries free I would like to share with your readership a simple clinical aid which I have been using for some time to help draw attention to the risks that erosion could be taking place so that parents can be forewarned. I call it the 'Five Circle Test'; with the Olympics approaching it seems an appropriate time to share it. When a child is being examined, assess the molars. As each cusp erodes a circle will appear on the cusp tip. If one circle is present (Fig. 1) less erosion has taken place than if the maximum number of five circles has appeared (Fig. 2) - hence the name. The test can be applied to both deciduous and adult molars and is an easy way of showing a parent what is happening and recording it in the patient's notes. Another useful aid is to move a gloved finger behind the upper incisor teeth tips looking for translucency (Figs 3-4).

M. Lowey

Stavanger, Norway DOI: 10.1038/sj.bdj.2010.57

\section{THE TIPPING POINT?}

Sir, in the editorial The artful science of politics (BDJ 2009; 207: 405) you highlighted the disconnect between evidence-based science and regulatory dogma as it affects the medical professions.

Sadly it is much easier to introduce new legislative and regulatory decisions than it is to remove them. When there are so many administrative matters to deal with, it may seem easier to leave things as they are rather than to unpick them - particularly if the make up of the team that originally introduced the changes has itself changed, possibly several times, since the original decision was made.

Only by periodically reviewing the decisions made on behalf of society can we be sure that they have continuing validity. This is done by assessing the decision against the evidence which has accumulated since its introduction. In addition any changes in the prevailing circumstances can also be taken into consideration.
In the case of dentists living with HIV disease the present regulations in the UK are well overdue for removal. Not only is there an international evidence base (29 look-back exercises in the UK alone) that fails to show transmission of HIV to patients in the dental setting but there have been two major developments since the regulations were first imposed by the Department of Health in response to the bizarre (and still unresolved) transmission that occurred to five dental patients treated by $\mathrm{Dr}$ Acer in Florida in the late 1980s. The Department is advised on such matters by the UK Advisory Panel for Healthcare Workers Infected with Bloodborne Viruses (UKAP).

The two developments are:

- Anti-retroviral therapy which is effective in lowering the viral level in those with HIV

- Markedly improved infection control standards adopted as standard for all dental patients.

The significance of this was recognised in the Beijing declaration ${ }^{1}$ issued last year at the 6th World Workshop on Oral Health and Disease in AIDS which states,

'The evidence now supports the view that Oral Health Care Professionals with HIV do not pose a risk of transmission to patients in the dental setting. They can continue a career in clinical practice, provided that the following criteria are met:

1) The individual is under ongoing care of a suitably qualified HIV Health Care Professional

2) The individual remains aware of their health status and acts appropriately

3) Standard Infection Control is observed

4) Scientific evidence related to HIV transmission will continue to be reviewed.'

This consensus statement was delivered by an international group of doctors and dentists, who also work with patients living with HIV/AIDS, following a week of debate that considered the available evidence base, back in April 2009. In the UK, things move more slowly. 
Following the same debate that was raised in this publication in October 2006 (BDJ 2009; 201: 497-499) UKAP has started, but has yet to complete, a review of the evidence as it affects HIV+ dentists; the project will take a little longer since it has now been expanded to consider all healthcare workers and other bloodborne diseases. The BDA stated its position in January 2007; 'with the appropriate drug treatment, physical barriers and universal cross infection control, the BDA recommends that HIV positive dentists be allowed to continue to work as normal in general dentistry'.

It comes as a breath of fresh air to read that the newly configured GDC should have acted on this matter in such a very positive way during their first months of operation. Following a debate by the Registration Committee, the following statement was published (November 2009 - Live regulatory policy issues; items 83-92).

Paragraph 92 states,

'With the Beijing declaration in place, the GDC may experience considerable difficulty (if not embarrassment) in seeking to pursue a case alleging impaired fitness to practise against an individual continuing to practise clinically (thus contravening extant Department of Health guidance) whilst being diagnosed with HIV.'

The realisation that, based on the present evidence, it would be discriminatory to perpetuate the present prohibition of HIV+ oral healthcare professionals who want to follow their chosen career has already been accepted by half of Europe, Australia and the USA.

Let us hope that at last we have reached the tipping point here in the UK and that 2010 holds a brighter future for those dentists and future dental students who would otherwise sacrifice their career because of the present inertia within the Department of Health and those who advise it.

D. Croser London

1. Proceedings of the 6th World Workshop on Oral Health and Disease in AIDS. J Dent Res (in press). The statement is available here: http://www. hivdent.org/_International_/2009/BEIJING DECLARATION_2009.htm

DOI: 10.1038/sj.bdj.2010.58

\section{VIOLENCE-FREE}

Sir, I recently had a case which was unique to me in 35 years in practice. A colleague was approached by a patient who was concerned about receiving dental treatment. The patient hadn't attended for some years as firstly he felt he had been poorly treated in other practices and secondly he had a history of lashing out at the dentist if he was hurt. As the gentleman in question is 6' 5" tall, 18.5 stones in weight and a close personal support specialist this was a consideration for both sides.

My colleague saw and spoke with the patient and managed to set him somewhat more at his ease and I was asked by both parties to consult with a view to providing sedation during treatment. Treatment was successfully provided under sedation (including large restorations and extraction) with both dentist and patient having a comfortable and violence-free time.

I have to say that this is the first time I have been asked to sedate someone because they were more worried about hurting the dentist than being hurt themselves.

\section{A. J. Caen \\ By email \\ DOI: 10.1038/sj.bdj.2010.59}

\section{OCCUPATIONAL STRESS}

Sir, the introduction of the new NHS dental contract in 2006 appears to have had a significant effect upon dentists working within NHS Primary Dental Care. It was widely reported by the media that this resulted in a marked increase in the levels of occupational stress experienced by GDPs.

Occupational stress was researched by the BDA in May 2005, before this new contract was introduced, and those results indicated that a high occupational stress level already existed.

Dentists' Provident is a company that provides support for dentists and their families affected by illness and our analysis of recent claims to them for stress-related illnesses show these have increased overall. For the years 2007 and 2008 the figures are shown in Table 1 (figures for earlier years are not available).

The consequences for those GDPs so affected cannot be calculated but there have been anecdotal reports of increasing depressive illness, marriage breakdown, alcohol/drug abuse and suicide. The concurrent credit crisis is likely to have been a contributory factor. For those who try to continue in practice, poor performance and a serious lack of pleasurable job satisfaction is reported.

These preliminary observations suggest that the 2006 new contract may have been associated with increased dissatisfaction, and that provision of NHS Primary Dental Care is in jeopardy. The reduction in claims for the 20-39 agegroup may reflect a feature of a small sample population, but does show that occupational stress is seen very early in the years following qualification and then develops progressively.

We are concerned as to attraction of future dental students to the profession unless significant improvements result from the changes that could flow from reviews such as the NHS Dental Services in England and recovery of the economy.

R. W. Matthews

M. I. Matthews

C. Scully CBE

By email

DOI: 10.1038/sj.bdj.2010.60

\begin{tabular}{|c|c|c|c|c|}
\hline YEAR & Age $20-39$ & Age $40-59$ & Age $60+$ & TOTAL \\
\hline 2007 & 18 & 67 & 13 & 98 \\
\hline 2008 & 14 & 74 & 20 & 108 \\
\hline$\%$ change in claims & -22 & +10 & +54 & +10 \\
\hline
\end{tabular}

